\title{
Analysis of Epidemic Characteristics and Related Pathogenic Factors of 2726 Cases of Pityriasis Alba
}

\author{
Yongmei Lv (D, Yamei Gao, Na Lan, Minghui Sun, Chenchen Zhang, Jing Gao, Na Wang, Xingyu Yang \\ Departments of Dermatology, The Second Affiliated Hospital of Anhui Medical University, Hefei, Anhui, 23060I, People's Republic of China \\ Correspondence: Yongmei Lv, Department of Dermatology, The Second Affiliated Hospital of Anhui Medical University, No. 678 Furong Road, Hefei \\ Economic and Technological Development Zone, Hefei, Anhui Province, 23060I, People’s Republic of China, Email yongmei_Iv830@I63.com
}

\begin{abstract}
Purpose: To understand the distribution characteristics of onset time, onset age and gender of pityriasis alba (PA) patients in the dermatology clinic of our hospital and to further explore the pathogenesis of the disease to provide a scientific basis for the prevention and treatment of this disease.

Patients and Methods: The clinical data of 2726 outpatients with PA diagnosed for the first time from January 2016 to December 2020 were collected and descriptively analyzed.

Results: The number of patients with PA was less from January to March. The peak was reached in July and August. The number of cases affected by the COVID-19 epidemic in 2020 was significantly lower than that in previous years. Furthermore, the onset age of the patients ranged from 0 to 64 years old, and the median age of the total population was $7(3,13)$ years old, including 1566 males $(57.45 \%)$ and 1160 females $(42.55 \%)$. The ratio of male to female was $1.35: 1$. The number of male patients before 18 years old was higher than that of female patients, especially in the high paroxysmal age group.

Conclusion: PA can be seen all the year round, and the onset peak of the disease is from July to August every year. It occurs frequently at the age of 1 to 14 before puberty. In the season of high incidence of PA, the protection from sun and moisture retention should be strengthened for infants and adolescents.
\end{abstract}

Keywords: pityriasis alba, epidemic characteristics, pathogenic factors

\section{Introduction}

Pityriasis alba (PA) is a multiple, round or oval with a few scales of hypopigmented spots. The size of the lesions is coinsized patches between $0.5-3 \mathrm{~cm} .{ }^{1-3}$ The damage can be gradually enlarged. The basal inflammation is mild or deficient. PA often occurs in the face, neck, extension of the upper arm and upper trunk of the child, and skin lesions can affect the appearance, especially in individuals with dark skin, the lesions look more obvious. ${ }^{1}$ Abnormal pigmentation often causes psychological discomfort to some children and parents, and some parents even worry that their children are suffering from vitiligo and bring their children to see a doctor anxiously. ${ }^{1,2}$ However, the disease has not attracted much attention from medical researchers due to its low harm, good prognosis and self-limiting. ${ }^{4-6}$ The aetiology of PA is not well established. ${ }^{1,2}$ In recent years, it has been considered that the disease is a subtype of atopic dermatitis that is related to specific allergic constitutions. ${ }^{1,4}$ Some studies have shown that the disease may be related to wind blowing, sun exposure, strong sunlight, soap stimulation, fungi, viruses, intestinal parasite infection, etc. ${ }^{6-8}$ Likewise, some scholars believe that the onset may be related to individual physiological skin metabolic problems, such as the temporary lack of certain trace elements and vitamins. ${ }^{2,8-10}$ In particular, the lack of zinc and calcium will indirectly affect the formation of pigments. In addition, it cannot be excluded that the disease is related to malnutrition and vitamin deficiency caused by many children anorexic to meals, vegetables or whole grains, but addicted to snacks, light snacks or having partiality for a particular kind of food. Some scholars believe that the occurrence of the disease may be related to personal hygiene habits (such as skin dryness caused by regular bathing, etc.). ${ }^{7}$ 
In order to understand the distribution characteristics of onset time, age and gender of PA outpatients in our hospital in recent years and to provide a scientific basis for effective prevention and control of the disease and further study of its pathogenesis.

\section{Patients and Methods}

\section{Research Object}

Statistical analysis of the patients with PA treated for the first time in our hospital from January 2016 to December 2020.

\section{Research Methods}

Using the statistical report of the outpatient electronic system of our hospital to collect the general data of patients with PA who visited the hospital from January 2016 to December 2020. When the same patient comes for many times, if the diagnosis is the same, it will be counted according to the time of the first visit. Finally, we counted a total of 2726 all PA patients who meet the above criteria with PA in the recent 5 years, ranging in age from 2 months to 64 years old. A retrospective method was adopted in this study. The protocol complied with the ethical guidelines of the Declaration of Helsinki and was approved by the Second Affiliated Hospital of Anhui Medical University's ethical committee. Parents or legal guardians have provided informed consent for the patients under 18 years of age after the research program has been explained. Moreover, descriptive epidemiological methods were used to analyze the distribution characteristics of onset time, onset age and gender of the disease.

\section{Statistical Analysis}

Excel 2007 worksheet (Microsoft, Redmond, WA, USA) was used to input case information for data collation, and Statistical Package for Social Science (SPSS) 11.5 statistical software (SPSS Inc., Chicago, IL, USA) was used for data statistics. Shapiro-Wilk test was used to test the normal distribution of the continuous data. Variables satisfying the normal distribution were described by mean \pm standard deviation (SD), otherwise, they were presented as the median (interquartile range, IQR). The categorical variables in this study were described as frequencies and percentages, and chisquare test was further used to compare their distribution differences. A two-sided $\mathrm{P}<0.05$ indicates that the difference is statistically significant.

\section{Results}

\section{Time Distribution Characteristics of the Number of Patients with PA}

In last 5 years, with the change of months, the number of patients with PA was relatively less from January to March, increased from April, reached the peak in July and August, and decreased obviously after September. However, there was still a relatively high incidence from September to December, as shown in Table 1 and Figure 1.

From 2016 to 2019, the number of patients per year has not changed much, with an average of approximately 600 patients. In 2020, due to the impact of the COVID-19 epidemic, the number of patients decreased significantly, as shown in Table 2 and Figure 2, with 347 people throughout the year.

Table I Monthly Statistical Data of the Outpatients with PA from January 2016 to December 2020

\begin{tabular}{|c|c|c|c|c|c|c|c|c|c|c|c|c|c|}
\hline Year & Jan & Feb & Mar & Apr & May & Jun & Jul & Aug & Sept & Oct & Nov & Dec & Total \\
\hline 2016 & 21 & 10 & 10 & 24 & 62 & 74 & 81 & 87 & 44 & 51 & 52 & 40 & 556 \\
\hline 2017 & 24 & 9 & 26 & 48 & 68 & 79 & 95 & 114 & 39 & 47 & 43 & 44 & 636 \\
\hline 2018 & 23 & 24 & 15 & 40 & 71 & 80 & 91 & 67 & 47 & 49 & 55 & 47 & 609 \\
\hline 2019 & 27 & 17 & 13 & 22 & 43 & 50 & 75 & 86 & 44 & 58 & 77 & 67 & 579 \\
\hline 2020 & 18 & 0 & 4 & 14 & 16 & 33 & 43 & 36 & 35 & 50 & 48 & 49 & 346 \\
\hline Total & 113 & 60 & 68 & 148 & 260 & 316 & 385 & 390 & 209 & 255 & 275 & 247 & 2726 \\
\hline
\end{tabular}




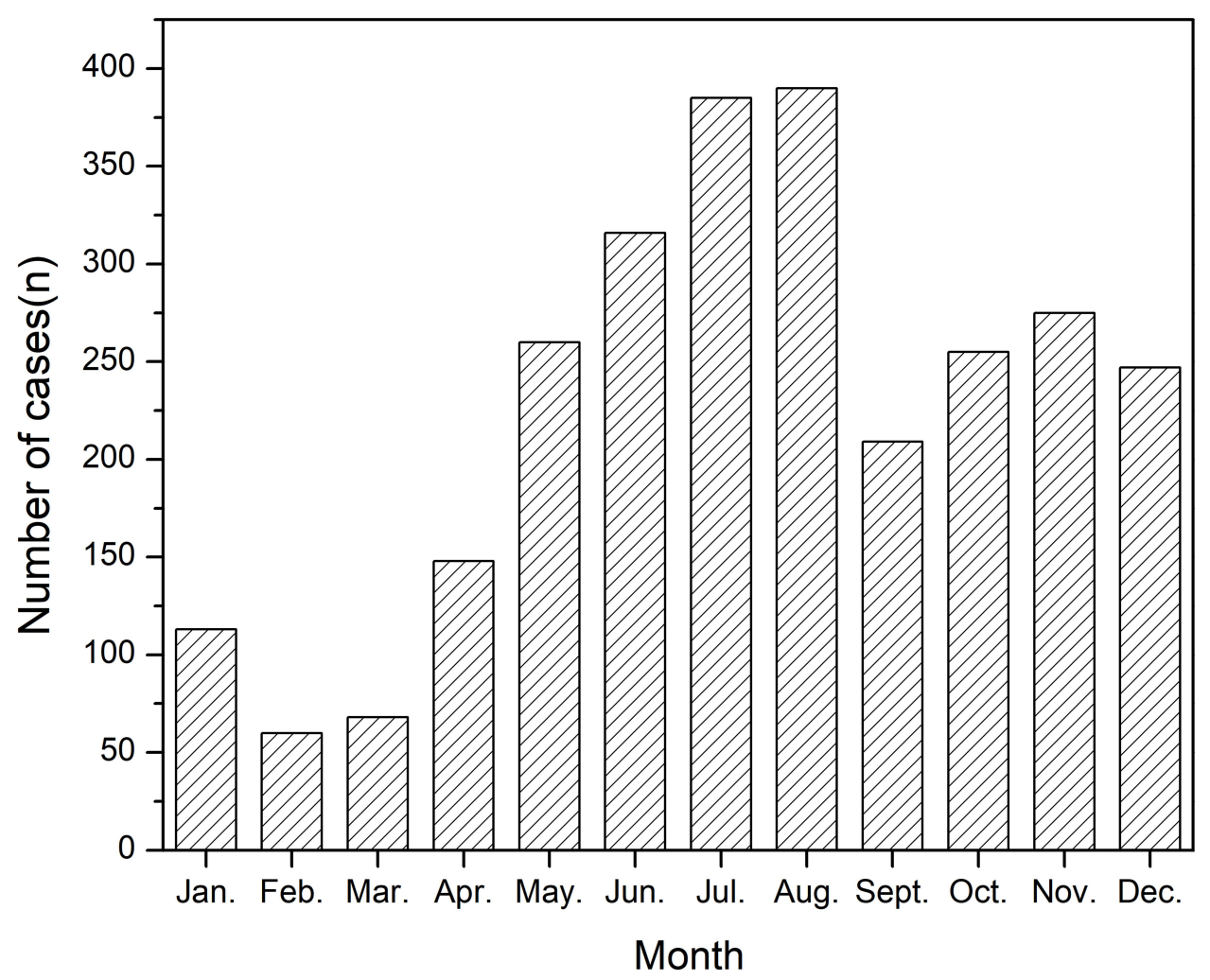

Figure I The average monthly number of outpatients with PA from January 2016 to December 2020.

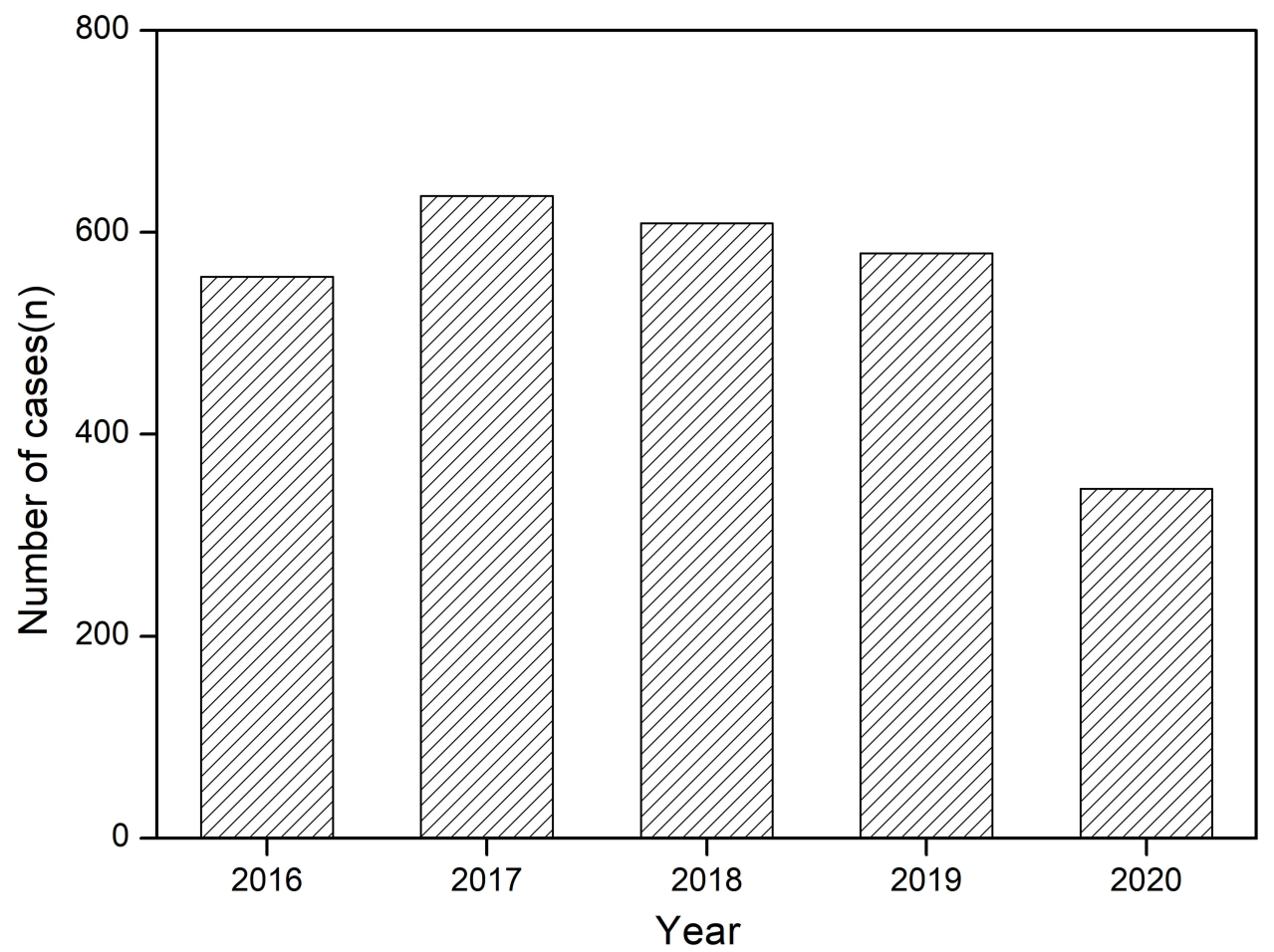

Figure 2 The average yearly number of outpatients with PA from 2016 to 2020. 


\section{Analysis of Age Distribution Characteristics of Patients with PA}

In the last 5 years, the onset age of patients with PA ranged from 2 months to 64 years old. The median age of the total population was $7(3,13)$ years old, including 1566 males $(57.45 \%)$ with a median age of $6(3,11)$ years, and 1160 females $(42.55 \%)$ with a median age of $7.5(3,20)$ years old. The ratio of males to females was $1.35: 1$ and the proportion of males was higher than that of females. There was no statistical significance in the age distribution of males and females $(\mathrm{P}=0.157)$. What's more, there were only 48 cases of infants less than 1 year old within 5 years accounting for $1.8 \%$ of the total. The disease mainly occurred in people aged 1 to 14 years old, accounting for $77.2 \%$ of the total. Exactly, the number of patients aged 1-9 years old remained at a high level, with more than 110 cases per year in each age group, especially in children aged 1-5 years old, with an average of more than 200 cases per year in each age group. The number of children after 10 years old decreased significantly, especially after 15 years old, and the total number of patients in each age group rarely exceeded 5 cases per year. There were few cases in the age class after the age of 41 , and the total number of cases within 5 years was 50 , accounting for $1.8 \%$ of the total. As is shown in Figure 3 .

\section{Analysis of Gender Distribution Characteristics of Patients with PA}

The number of male patients before 18 years old was higher than that of female patients, especially before 14 years old. However, the incidence was lower in both males and females after 18 years old, and the morbidity number of females aged 18 to 36 years old was slightly higher than that of males (see Figure 4).

\section{Discussion}

Our survey found that patients with PA can be seen throughout the year, whereas the number of cases varies with the month. Specifically, the consultation rate of patients was lower in the cold winter from January to March, and the incidence peaked in July and August. This phenomenon is considered to be related to the strengthening of moisturizing protection in winter and the longer exposure time of sunlight in summer. The correlation between sunlight exposure time and intensity, environmental humidity, wind blowing, skin moisturizing and so on, and the disease has been confirmed by many experts. ${ }^{11,12}$ Further analysis found that the number of patients increased gradually from April every year. This is

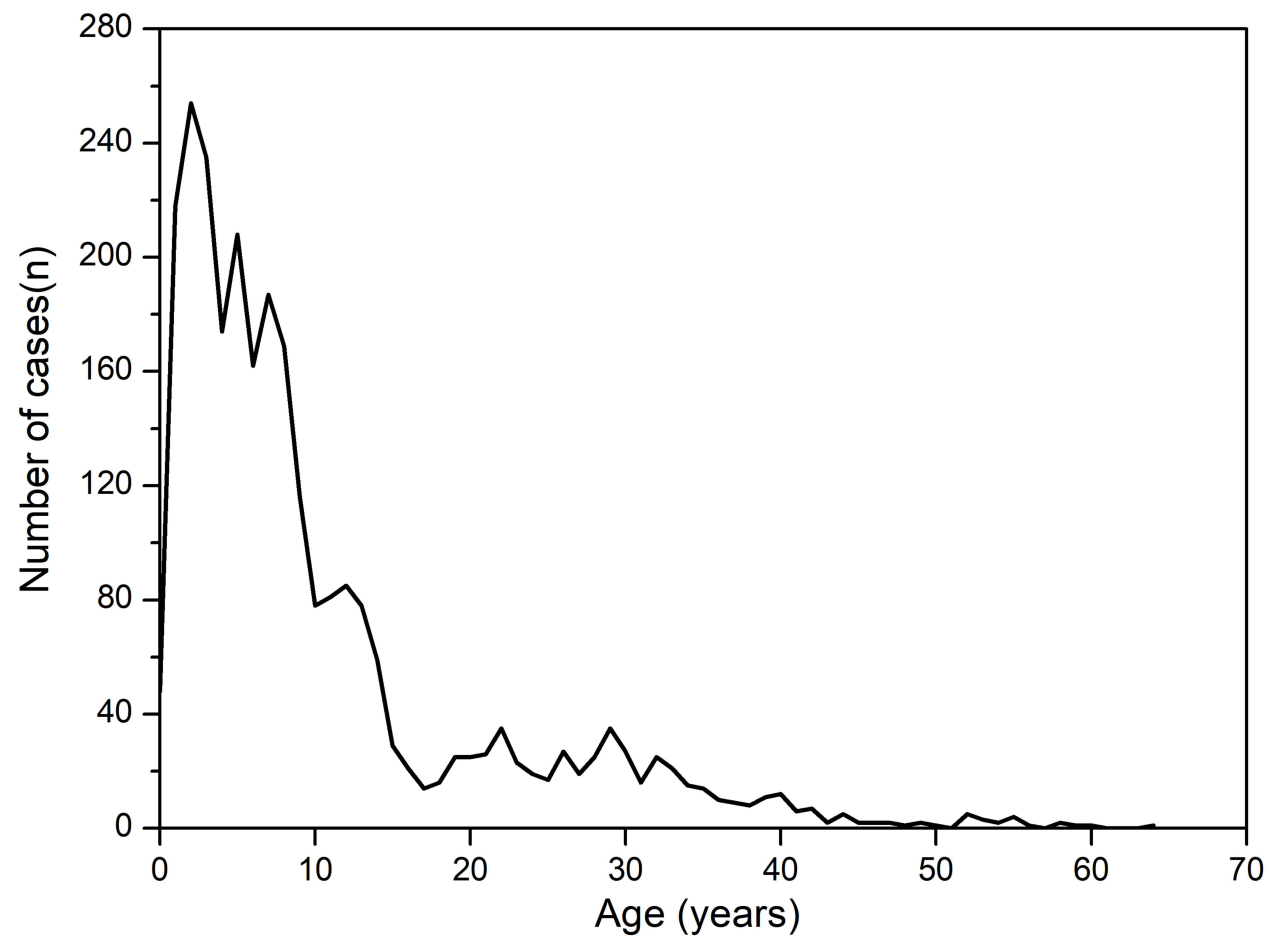

Figure 3 The line graph distribution of the outpatients with PA correlation with the onset age from January 2016 to December 2020. 


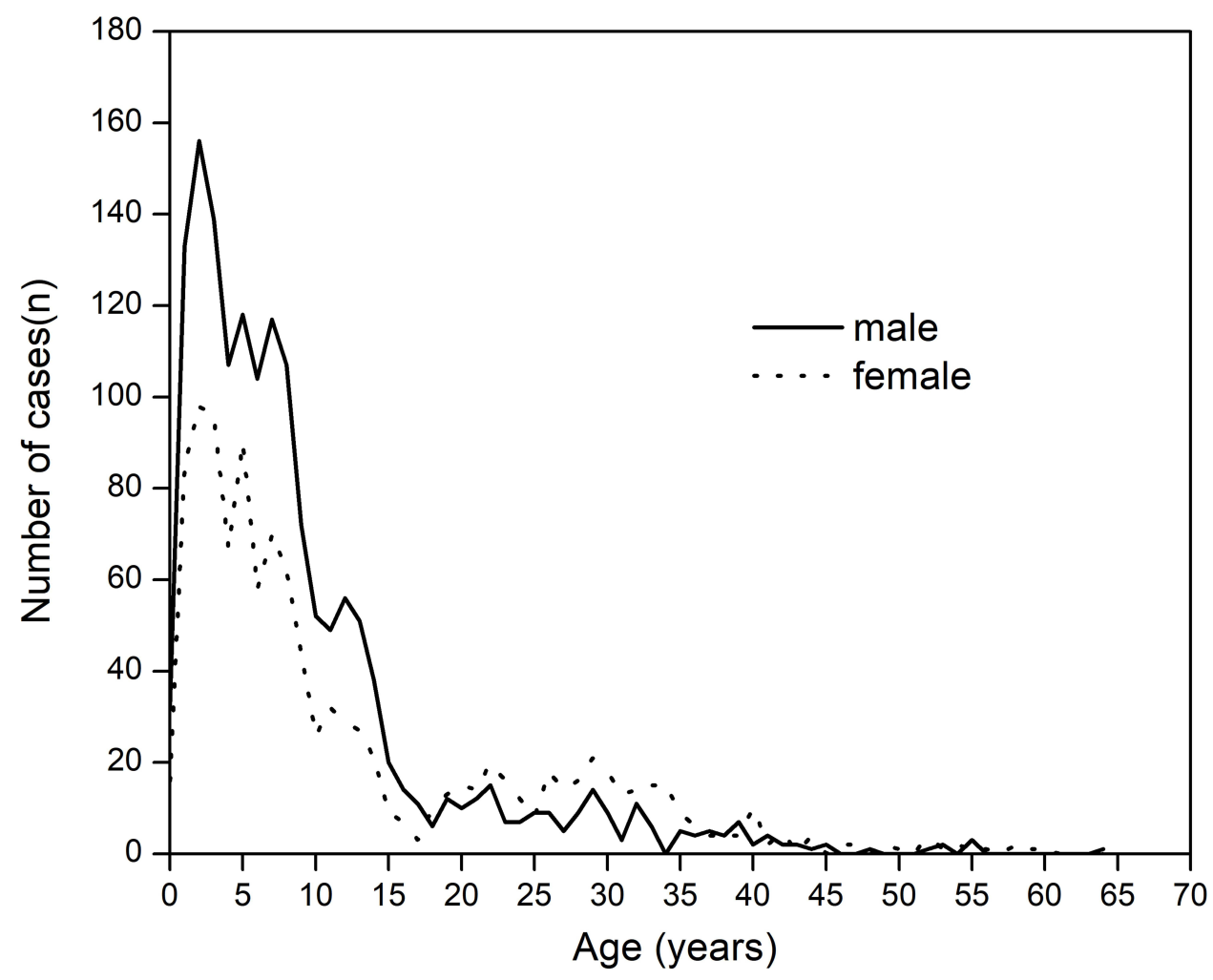

Figure 4 The line graph distribution of gender of the outpatients with PA correlation from January 2016 to December 2020.

related to the increase in the number of people going out for hiking after the weather is warmer, and is related to the factors relevant to the wind and the sun. The incidence reaches its peak in July and August, which is related not only to the overexposure to sunlight, ${ }^{7}$ but also to the direct effect of ultraviolet radiation on melanocytes. This direct radiation reduces the number and activity of melanocytes and leads to hypopigmentation of the epidermis. The causes of the peak are not excluded that this time period is during the summer vacation of primary and secondary school students, but also there are more opportunities to be exposed to ultraviolet rays. Moreover, it is also convenient for parents to arrange the time to see a doctor during the holidays. The number of patients decreased significantly with the arrival of the school season in September, and there was still a higher incidence in the dry autumn and winter from September to December, which was considered to be related to dry climate, wind blowing, lack of moisturizing and skin care, etc. The number of people is the lowest in the cold winter and spring, especially in February and March, which is related not only to the decrease in ambient temperature and the decrease in sun exposure but also to the increase of local moisturizing consciousness in winter. In short, our investigation shows that the number of patients with the disease changes significantly with the seasonal changes, although it has also been reported that there is no clear correlation between the seasonal changes and the occurrence of the disease. ${ }^{8}$ In addition, the number of patients per year from 2016 to 2019 was roughly similar, and the number of patients with PA in 2020 was significantly lower than that in previous years. We found that the decline in the number of people going to the hospital in 2020 was caused by the impact of the COVID-19 outbreak, state control of going out, and travel restrictions, where sun exposure was significantly reduced. This phenomenon also confirmed that there is a great correlation between sun exposure and the incidence of the disease. However, there are some limitations on accurate onset time in our research, and the uncertainty of delaying the time of seeing a doctor after the onset of the disease, which may lead to a deviation in the accuracy of the investigation.

Our study also found that the disease often occurs in children aged 1 to 14 years old, and the number of children aged 1 to 9 years old remains at a high level, especially in children aged 1 to 5 years old, and the number of adolescent cases decreases gradually after 15 years old. Adults after the age of 41 rarely get sick. This is slightly different from many reports at the age of 3-16 years old. ${ }^{12-14}$ Related studies have analysed the causes of prepubertal children with the disease, which is related to the 
fragile skin barrier and dry skin of children. Some studies have shown that the thickness of the epidermis after puberty is significantly higher than that in infancy, preschool age and school age groups. ${ }^{15}$ Furthermore, the skin is protected by sebaceous membranes after puberty, and the incidence of puberty and adults is low. It is also related to various factors such as the dryness of the skin, because the skin is more resistant to sunlight, wind stimulation and soap cleaning. Further analysis shows that the disease tends to occur in preadolescent children in China, which may be related to the fact that most parents encourage their children to exercise outdoors, consciously let their children bask in the sun, promote calcium absorption ${ }^{16}$ and prevent myopia. ${ }^{17}$ In addition, children of this age have no awareness of sun protection when playing and lack of corresponding sun protection measures. Of course, some children experience genetic damage to the skin barrier, dry skin caused by improper skin bathing, and allergic skin diseases caused by external allergen irritation. For example, children with atopic dermatitis often have PA. Related experts in PA and atopic dermatitis also agree that the two are closely related to each other. ${ }^{1,4,5,12,18,19} \mathrm{PA}$ is also an important reference for the diagnosis of atopic dermatitis as a common concomitant disease. Some scholars believe that even if there are no symptoms of atopic dermatitis when suffering from PA, it is possible to develop atopic dermatitis later in life. ${ }^{2,7} \mathrm{PA}$ in adolescence and adulthood may also be hypopigmented patches left after inflammatory skin diseases such as dermatitis and eczema. ${ }^{19}$

Our study also confirmed that the incidence of male patients is higher than that of female patients before the age of 18, and the incidence is significantly higher than that of females even before the age of 14, which is the high paroxysmal age for PA. We think that this was related to the fact that most boys are lively, active and have more outdoor activities. They were often exposed to wind and the sun, and neglect skin sunscreen and moisturizing care. ${ }^{20}$ This is also basically consistent with the research of Blessmann and O'Farrell et al ${ }^{10-12}$ The phenomenon that the incidence of PA in females was slightly higher than that in males after adulthood may be related to the fact that generalized PA is more common in females, which needs further clinical verification. ${ }^{20}$

To sum up, this study used a large sample of patients with PA to analyze the distribution characteristics of onset time, age and gender of the disease to provide a scientific reference for effective education and prevention of PA. However, since it is not possible to determine the corresponding number of exposed populations, it is not possible to calculate the incidence. Therefore, this work has some limitations and needs in-depth study in the near future.

In summary, PA could be seen all year round, and the peak of the disease occurred from July to August every year. It often occurs between the ages of 1 and 14 before adolescence. In the high incidence season of PA, infants and young people should strengthen the sunscreen and moisturizing.

\section{Acknowledgments}

This work was supported by the "Clinical Research Cultivation Program" of the Second Affiliated Hospital of Anhui Medical University (2020LCYB12) and the Research Funds from China Tobacco Anhui Industrial Co., Ltd. (No.2021132). The team leader of the former is the corresponding author of this paper and the funding of the latter is also one of the authors of this article.

\section{Author Contributions}

All authors made a significant contribution to the work reported, whether that is in the conception, study design, execution, acquisition of data, analysis and interpretation, or in all these areas; took part in drafting, revising or critically reviewing the article; gave final approval of the version to be published; have agreed on the journal to which the article has been submitted; and agree to be accountable for all aspects of the work.

\section{Disclosure}

The authors report no conflicts of interest in this work.

\section{References}

1. Zaynoun ST, Aftimos BG, Tenekjian KK, Bahuth N, Kurban AK. Extensive pityriasis alba: a histological histochemical and ultrastructural study. $B r$ J Dermatol. 1983;108(1):83-90. doi:10.1111/j.1365-2133.1983.tb04582.x 
2. In SI, Yi SW, Kang HY, Lee ES, Sohn S, Kim YC. Clinical and histopathological characteristics of pityriasis alba. Clin Exp Dermatol. $2009 ; 34$ (5):591-597. doi:10.1111/j.1365-2230.2008.03038.x

3. Al-Mutairi N, Al Hadad A. Efficacy of 308-nm xenon chloride excimer laser in pityriasis alba. Dermatol Surg. 2012;38(4):604-609. doi:10.1111/ j.1524-4725.2011.02223.x

4. Sandhu K, Handa S, Kanwar AJ. Extensive pityriasis alba in a child with atopic dermatitis. Pediatr Dermatol. 2004;21(3):275-276. doi:10.1111/ j.0736-8046.2004.21321.x

5. Pinto FJ, Bolognia JL. Disorders of hypopigmentation in children. Pediatr Clin North Am. 1991;38(4):991-1017. doi:10.1016/S0031-3955(16) 38164-0

6. Carneiro FRO, Do Amaral GB, Mendes MD, Quaresma JAS. Tissue immunostaining for factor XIIIa in dermal dendrocytes of pityriasis alba skin lesions. An Bras Dermatol. 2014;89(2):245-248. doi:10.1590/abd1806-4841.20142201

7. Weber MB, de Avila LGS, Albaneze R, de Oliveira OLM, Sudhaus BD, Cestari TF. Pityriasis alba: a study of pathogenic factors. J Eur Acad Dermatol Venereol. 2002;16(5):463-468. doi:10.1046/j.1468-3083.2002.00494.x

8. Bassaly M, Miale A, Prasad AS. Studies on pityriasis alba. a common facial skin lesion in Egyptian children. Arch Dermatol. 1963;88(3):272. doi:10.1001/archderm.1963.01590210030004

9. Wat H, Dytoc M. Off-label uses of topical vitamin D in dermatology: a systematic review. J Cutan Med Surg. 2014;18(2):91-108. doi:10.2310/ 7750.2013 .13109

10. Elesawy FM, Akl EM, Halim WAA. Zinc has a role in pathogenesis of pityriasis alba. Indian J Paediatr Dermatol. $2020 ; 21(3): 178-183$.

11. Ofarrell NM. Pityriasis alba. Arch Dermatol. 1956;73(4):376-377. doi:10.1001/archderm.1956.01550040070010

12. Jadotte YT, Janniger CK. Pityriasis alba revisited: perspectives on an enigmatic disorder of childhood. Cutis. 2011;87(2):66-72.

13. Leung AKC, Feingold M. Picture of the month: pityriasis alba. Am J Dis Child. 1986;140(4):379-380.

14. Wells BT, Whyte HJ, Kierland RR. Pityriasis alba: a ten-year survey and review of the literature. Arch Dermatol. 1960;82(2):183-189. doi:10.1001/ archderm.1960.01580020025003

15. Zhang Y, Tang Y, Quan X, et al. Preliminary study of the ultrasonic measurement of thickness of skin in children. Chin J Burns. 2007;23 (5):183-184.

16. Wang H, Shi H, Chang L, et al. Association of blood lead with calcium, iron, zinc and hemoglobin in children aged 0-7 years: a large populationbased study. Biol Trace Elem Res. 2012;149(2):143-147. doi:10.1007/s12011-012-9413-x

17. Jin JX, Hua WJ, Jiang X, et al. Effect of outdoor activity on myopia onset and progression in school-aged children in northeast China: the Sujiatun Eye Care Study. BMC Ophthalmol. 2015;15:73. doi:10.1186/s12886-015-0052-9

18. Darnell TFB. Relationship of pityriasis alba to eczema. South Med J. 1962;55(2):183. doi:10.1097/00007611-196202000-00018

19. Miazek N, Michalek I, Pawlowska-Kisiel M, Olszewska M, Rudnicka L. Pityriasis alba common disease, enigmatic entity: up-to-date review of the literature. Pediatr Dermatol. 2015;32(6):786-791. doi:10.1111/pde.12683

20. Fenner J, Silverberg NB. Skin diseases associated with atopic dermatitis. Clin Dermatol. 2018;36(5):631-640. doi:10.1016/j. clindermatol.2018.05.004

Clinical, Cosmetic and Investigational Dermatology is an international, peer-reviewed, open access, online journal that focuses on the latest clinical and experimental research in all aspects of skin disease and cosmetic interventions. This journal is indexed on CAS. The manuscript management system is completely online and includes a very quick and fair peer-review system, which is all easy to use. Visit http://www. dovepress.com/testimonials.php to read real quotes from published authors.

Submit your manuscript here: https://www.dovepress.com/clinical-cosmetic-and-investigational-dermatology-journal 